This year's program also proved one of the most successful in convincing students to apply to graduate programs in political science. More than half of the students have indicated that they are serious about pursuing graduate studies.

Because of its rigorous program, the Institute's scholars have seen great success. A number of alumni have received master's and Ph.D. degrees and three have been named assistant professors. This year, alumnae Yvette Alex-Assensoh, assistant professor at Indiana University; Khalilah Brown, a graduate student at Ohio State University; Andra Gillespie, a graduate student at Yale University; Monique Lyle, a graduate student at Duke University; and Carmen Huerta, an incoming graduate student at Rice University, returned to share stories of how participation in the Institute has benefitted them.

The Institute's namesake, Ralph Bunche, a 1934 Harvard University graduate, was the first African American to receive a doctorate in political science and the first to serve as the president of the American Political Science Association. In
1950, he also became the first African American to receive the Nobel Peace Prize.

The Institute has been held at the University of Virginia for the past five years and will be moving to Duke University for the summer of 2001. The program is supported financially by grants from the National Science Foundation and the American Political Science Association and receives substantial support from the host institution.

\section{9-2000 APSA Minority Fellows Choose Graduate Schools}

Since 1969, APSA's minority Fellows Program has pursued the goal of encouraging minority participation in Ph.D. programs. African American, Latina/o, and Native American students who demonstrate an interest in teaching and display research potential in political science are encouraged to apply for conditional one-year grants to be used for graduate school tuition and fees. Funded or unfunded, the benefits fellows receive are innumerable. Being named an APSA minority fellow marks a student as an excellent

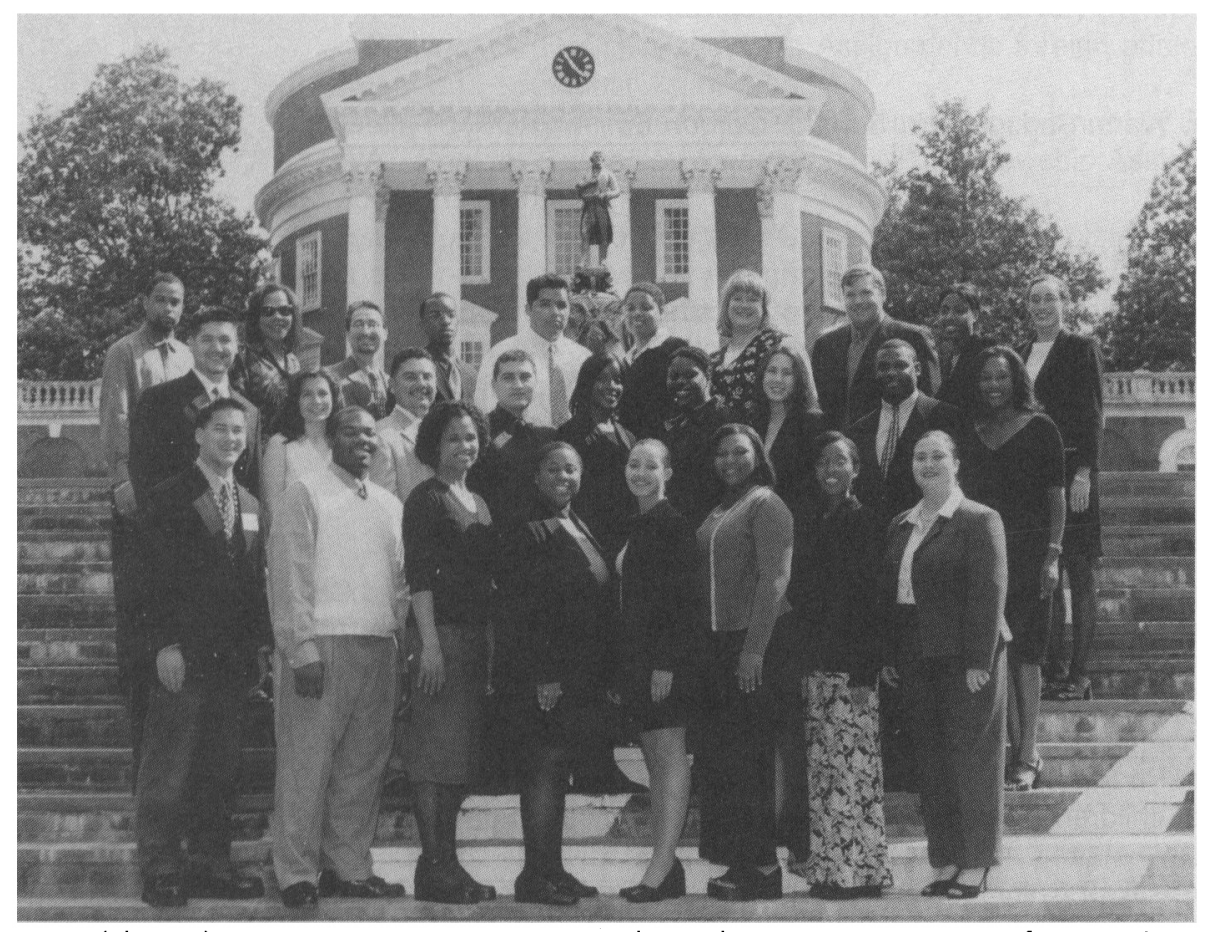

2000 Ralph Bunche Summer Institute participants. Back row (I to r): Byron Demery, Professor Paula McClain, Professor Paul Freedman, Jawad Towns, Adan Balboa, Renee Jeffries, Karon Coc (TA), Patrick McGuinn (TA), Beonica Rupert, and Vesla Weaver. Middle row (I to r): John Amaya, Stacy Nyikos (TA), Gabriel Sanchez, Nicholas (Sammy) Fernandez, Yashica Danridge, Deva Woodly, Tehama Lopez, J. Alan Kendrick (TA), and Alexis Clark (TA). Front row (I to r): Michael Tuncap, Elgin Rogers, Jr., lfika Oldwine, Shabazz Wilson, Angelique Douyon, Raasheja (Nicci) Page, Michelle Oby, and Gladys De La Cruz. Not pictured: Darby Morrisroe (TA). Photo by James Carpenter.

candidate for graduate study and the nation's top graduate programs often recruit minority fellows with offers of school-sponsored fellowships that include remission of tuition and fees, assistantships, and elevated stipends. More than 70 former minority fellows now hold Ph.D.s in political science.

\section{African-American Fellows}

\section{Eleanor Bright}

Fleming will attend Vanderbilt University, where she has been designated a University Graduate Fellow, a Dean's

Graduate Fellow,

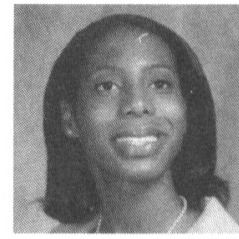

Eleanor Bright and the recipient of the Richard $\mathrm{B}$.

Bennett/Dorothy Compton Prize.

Ian Yusef Needham will attend the University of Chicago, where he will receive a fellowship.

DeAunderia Bryant will attend the University of Michigan with a fellowship

Aleshia M.

Dyson will attend

Georgia State

University, where she will receive an assistantship and a stipend

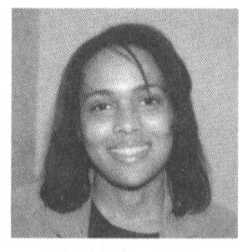

Aleshia Dyson

\section{Latino Fellows}

Jason Paul Casellas will attend Princeton University

\section{Carmen Huerta} will attend Rice University, where she has been granted a fellowship with remission of tuition and fees and a stipend.

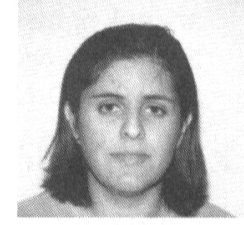

Natasha Borges

Carmen Huerta

Sugiyama will attend the University of Texas, Austin, where she received a teaching assistantship with remission of tuition and fees.

Adele Elizabeth Vigil will attend the University of California, San Diego, where she has been granted a fellowship with remission of tuition and fees.

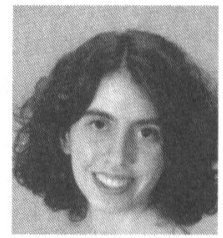

Adele Vigil 


\section{Political Science Fellows}

Robert G. Boatright, Ph.D. candidate, Univeristy of Chicago: Representative David Price (D-NC). Fellow Assignments: budget, commerce, campaign finance, science, social security, and energy.

Daniel Kaufman, Ph.D. candidate, University of California, San Diego: Representative Chartes B. Rangel (D-NY). Fellow Assignments: international drug policy and other Latin-American issues (immigration, trade, education).

Andrew Taylor, Assistant Professor, North Carolina University (William A. Steiger Fellow): Representative Christopher Shays (RCT). Fellow Assignments: budget, taxes, transportation.

Leslie McCollum, Ph.D. candidate, University of Oklahoma (Carl Albert Fellow): Representative Marge Roukema (R-NJ). Fellow Assignments: education.

Craig Williams, Ph.D. candidate, Univeristy of Oklahoma (Carl Albert Fellow): Representative Robert L. Ehnlich Jr. (R-MD). Fellowship Assignmnets: defense, veterans affairs, international relations, education.

\section{Journalism Fellows}

Tanya Ballard, Reporter, The Tennessean, Nashvile, TN (Knight Foundation Fellow): Representative Bobby Rush IL). Fellowship Assignments: census, immigration reform, education, transportation, workforce, welfare Jennifer Chaney, Entertainment Editor, Gazette Newspapers, Gaithersburg, MD (Knight Foundation Fellow): Representative Louise M. Slaughter (D-NY). Fellowship Assignments: women's issues, arts.

Hazel Trice Edney, John F. School of Government, Harvard Univeristy (Kight Foundation Fellow): Senator Edward M. Kennedy (D-MA). Fellowship Assignments: website articles on various issues.

Lynne Weil, European Correspondent, Catholic News Service, Rome (Congressional Quarterly Fellow) Fellowship Assignment: foreign policy.

Kim Wellman, News Director, KKPR AM-FM, Kearney, NE: Senator Chuck Hagel (R-NE). Fellowship Assignments: agricultural issues, ethanol, reserve program.

Chantal Blouin, Ph.D. candidate, University of Toronto (APSA-MCI WorldCom Fellow):Representative David Minge (D-MN). Fellowship Assignments: antitrust, telecommunications.

James Snider, Ph.D. candidate, Northwestern University (APSA-MCI WorldCom Fellow): Senator Ron Wyden (D-OR). Fellowship Assignments: information policy, telecommunications, electronic commerce.

\section{Hatfield Fellow}

Direlle Calica, Tribal Paralegal Specialist, Warm Springs, OR: Senator Gordon Smith (R-OR). Fellowship Assignment: Native American affairs.

\section{German Marshall Fellows}

Michael Kolkmann, Research AssistantPh.D. candidate, Univeristy of Potsdam, Berlin: Representative Rush Holt (D-NJ). Fellowship Assignments: foreign affairs, defense, environment, transportation, immigration, animal rights/wildlife, civil rights, women's issues, judiciary issues.

Simmone Stemm/er, Program Coodinator, Ph.D. candidate, Munich Univeristy, Munich: Representative Joseph Crowley (D-NY). Fellowship Assignments: foreign relations, global health, immigration.

\section{Ford Foundation Fellow}

Mohamed Kamal, John Hopkins University, The Nitze School of Advanced International Studies and Cairo University, Cario, Egypt: Representative Tom Sawyer (D-OH). Fellowship Assignments: international relations, education.

\section{Robert Wood Johnson Foundation Health Policy Fellows}

Charletta Guillory, Assistant Professor of Pediatrics, Baylor College of Medicine: Representative John B. Breaux (DLA). Fellowship Assignments: Medicare, children's health insurance, Medicaid, medical errors, Breast-Cervical Treatment Act, community health centers.

Sally Phillips, Associate Professor, University of Colorado School of Nursing: Senator Tom Harkin (D-IA). Fellowship Assignments: health, education, labor and pensions committee (Ryan White Care Act, Public Health, children's health bill, National Health Services Corporation).

Mark M. Rasenick, Professor, University of Illinois, Chicago: Senator Edward M. Kennedy (D-MA), Senate Committee on Health, Education, Labor, and Pensions. Fellowship Assignments: helath, science. 
David A. Russell, Assistant Dean of Clinical Affairs, Tufts University School of Dental Medicine: Senator Orrin G. Hatch (R-UT), Senate Committee on the Judiciary, Majority Staff. Fellowship Assignments: health, dental care, mental health.

Jeff Shornick, Clinical Associate Professor, University of Washington: Senator John D. Rockefeller IV (D-WV). Fellowship Assignments: Patient Bill of Rights, perscription drug proposals for Medicare, medical errors, stem cell research.

Karen Edison Zanol, Assistant Professor of Dermatology, University of Missouri, Columbia: Senator James M. Jeffords (R-VT), Senate Committee on Health, Education, Labor, and Pensions. Fellowship Assignments: safety net providers, community/migrant health centers, public hospitals, veterans healthcare issues, graduate medical education, tax credits, medical errors.

\section{Asia Foundation Fellow}

Chung Youl Lee, Expert Advisor, Tripartite Commission, Seoul, Korea: Representative Lleana Ros-Lehtinen (R-FL). Fellowship Assignments: trade policy, export controls, international investment policy, international financial institutions.

\section{Foreign Affairs Fellows}

John Abbatiello, Intelligence Operations Specialist, Defense Intelligence Agency: Joseph I. Lieberman (D-CT), Governmental Affairs Committee. Fellowship Assignments: federal government computer security legislation, federal government fiscal mismanagement, share-in-savings contracts, intelligence-law enforcement coordination.

$\mathrm{Kel}$ Jacon Britvec, Senior Intelligence Officer, Defense Intelligence Agency: Representative Debbie Stabenow (D-MI). Fellowship Assignments: judiciary, crime, immigration, foreign affairs, defense/national security, veterans affairs, Gulf War Syndrome, transportation.

R. Wes/ey Carrington, Assistant Public Affairs Officer, United States Information Agency: Senator Paul $D$. Wellstone (D-MN). Fellowship Assignments: foreign affairs.

Laban L. Coblentz, Special Assistant Public Affairs Officer, United States Information Agency: Senator Joseph I. Lieberman (D-CT), Govermmental Affairs Committee. Fellowship Assignments: intergovernmental relations, government information, internet.

Kim A. Cornwall, Chief Engineer, Central Intelligence Agency: Senator Conrad Bums (R-MT). Fellowship Assignements: telecommunications, defense, foreign relations, veteran affairs.

David Dorman, Chief Intelligence Analysis Branch, National Security Agency: Senator Chuck Hagel (R-NE). Fellowship Assignment: foreign policy.

Carter Edgeworth, Intelligence Office Geographer, Central Intelligence Agency: Senator Sam Brownback (R-KS). Fellowship Assignments: foreign affairs (Africa, Central/Latin America, Europe, East and Southeast Asia).

Katherine Hadda, International Economist, Department of State: Representative Jim McDermott (DWA), Senator Russell D. Feingold (D-WI). Fellowship Assignments: budget issues, back-stopping on trade, international crime.

Abigail Morales, Senior Program Officer, United States Information Agency: Representative Brad Sherman (D-CA). Fellowship Assignments: international relations.

Ken Moskowitz, Information Officer, United States Information Agency: Representative Nancy Pelosi (D-CA), Senator James $M$. Jeffords (R-VT). Fellowship Assignments: foreign affairs, trade.

John Michael Underriner, Special Assistant to the Bureau of African Affairs, Department of State: Senator Tom Harkin (D-IA), House International Relations Committee. Fellowship Assignments: international relations.

\section{Federal Fellows}

Gloria Anthony, Information Systems Security Analyst, National Security Agency: Representative Melvin L. Watt (D-NC). Fellowship Assignments: transportation, veterans affairs, agriculture, human rights, health.

Erastace N. Fields, Reactor Systems Engineer, Nuclear Regulatory Commission: Albert R. Wynn (D-MD). Fellowship Assignments: District of Columbia issues, education, nuclear waste, electric industry restructuring.

Hannah Patricia Marter, Management Staff for Office of Inspector General, National Reconnaissance Office: Senator Jack Reed (D-RI). Fellowship Assignments: health care, on line pharmaceuticals, organ donation/transplants, DoDI Breast Cancer Research Program.

Glen Sauer, Deputy Chief, Protective Services, National Security Agency: Senate Governmental Affairs Committee, Subcommittee on Intemational Security, Proliferation and Federal Services. Fellowship Assignments: terrorism, proliferation of weapons of mass destruction, information system protection initiatives, rising college tuition costs.

Cynthia Taylor, Physical Scientist, National Imagery and Mapping Agency, DOD: Representative Henry J. Hyde (R-IL).

Fellowship Assignments: international relations, defense, labor. 